\section{Cosmetic injectables under MPs' spotlight}

The British Dental Association has urged dentists to be appropriately protected, as MPs call on the government to end the "Wild West' of non-surgical beauty treatments.

Many patients seeking cosmetic treatment remain unaware of the degree of protection that is missing when seeking apparently similar treatment from an unregulated individual working from premises that have not been inspected by CQC. The All-Party Parliamentary Group on Beauty, Aesthetics and Wellbeing is calling on the government to address the need for regulation of such procedures and the associated injectable cosmetic agents, including appropriate insurance.

MPs spent a year on an inquiry and have made 17 recommendations following concerns that currently anyone can carry out any treatment, with minimal legal restrictions on who can provide them or what qualifications they must have to do so. The insufficient legal framework governing these treatments has left patients at risk and undermined the industry's ability to develop. Len D'Cruz Head of BDA Indemnity said: 'Our policyholders are indemnified for the use and administration of facial cosmetic injectable procedures above the lower border of the mandible. This is part of all Extra and Expert members' policy terms. This has been the case since the indemnity policy exclusively for BDA members was launched three years ago.

'The use of injectable cosmetic treatments in the peri-oral area is a useful adjunct to dental treatment when provided by a suitably trained dentist. In addition, your patients are protected by your indemnity arrangements and a process for managing complaints if anything should go wrong.

'The GDC considers these treatments within the scope of practice of dentists providing appropriate training has been undertaken?

\title{
Dates for the diary
}

Friday 17

September -

ONLINE Adult and

child safeguarding

Live, interactive

workshop, hosted on

Zoom, limited to a

small group

4 hours of CPD -

covers levels 1 and 2 in

safeguarding

Book online: bda.org/

training

Business essentials: what every practice owner needs to know

2-day course, London

Day 1: Friday 10 September

Day 2: Friday 8 October

Book online: bda.org/training

\section{Expansion of sugar tax a litmus test for government on prevention}

The British Dental Association has backed recommendations set in the second part of the Food Strategy to expand the sugar tax to cover foods high in sugar and salt.

The plan, developed by 'Food Tsar' Henry Dimbleby, advocates a sugar and salt tax on unhealthy food, which would direct some of the revenues to help get fresh fruit and vegetables to low-income families. The move would build on the success of the sugar levy, which prompted a fall in sales of high-tier sugary drinks - those containing

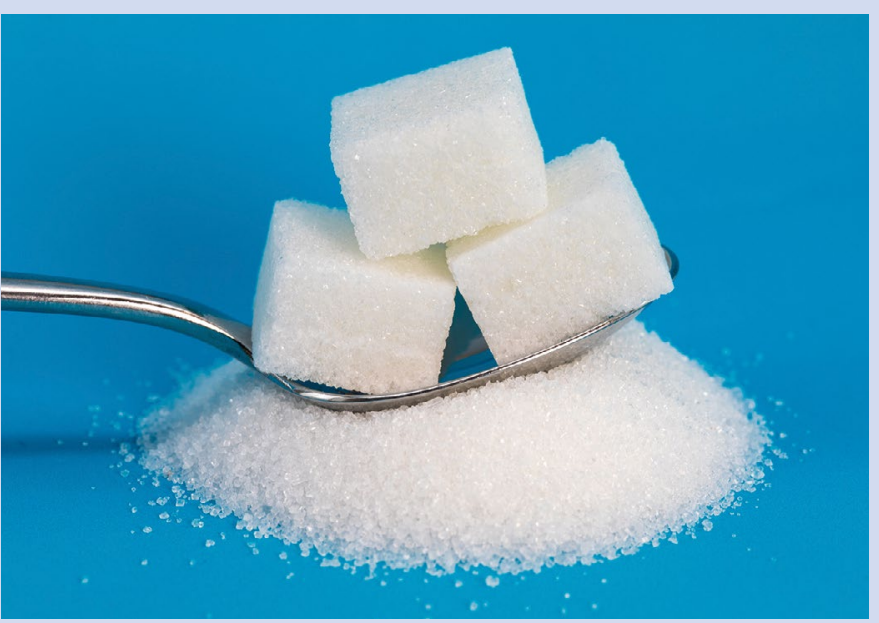

more than $8 \mathrm{~g}$ of sugar per $100 \mathrm{ml}$ - of $44 \%$ in its first year.

Tooth decay has been the number one reason for hospital admissions among young children in the UK, with the burden hitting those in the most deprived communities the hardest. The strategy notes children living in the UK's most deprived communities are 3 times more likely to have tooth decay at age 5 .

The BDA expects these inequalities to widen as a result of the COVID-19 pandemic. While dentist leaders have welcomed moves in the Health and Care Bill currently before parliament to aid the expansion of schemes such as water fluoridation, it says action needs to be part of a joined-up approach, addressing issues from access to services to food culture.

The BDA wants to see sustained government action to tackle diet-related disease like tooth decay as an integral part of ongoing work to confront obesity. It has also welcomed recommendations to expand eligibility for free school meals and extend the provision of programmes to tackle holiday hunger.

BDA Health and Science Chair Mick Armstrong said: 'If the government is serious about tackling preventable disease it must build on the ambition set out in this strategy. Over consumption of sugar causes both dental decay and obesity but dental disease is largely irreversible. That link should be made clear and more widely acknowledged.

'Ministers are making the right noises on prevention but need to take a joined-up approach. Willingness to build on the success of the sugar levy will be a litmus test.

'Every day dentists see how sugar fuels ill health and grotesque inequalities that are now set to widen. Ministers must show they are willing to act on root causes, and fix Britain's failed food culture.' 